\title{
The Retinal Atlas Editor: Lawrence A Yannuzzi 2010 928pp., Hardcover US\$299 ISBN: 978-0-7020-3320-9 Saunders Elsevier
}

\author{
Michael Ulbig
}

Received: 1 September 2010 /Accepted: 1 September 2010/Published online: 18 January 2011

(C) Springer-Verlag 2011

As the title notes, this is an atlas, which means that the pictures appear prior to the text. The title is concise and clear. In my opinion, this is absolutely the retinal atlas.

A hardback with 910 pages, subdivided into 15 chapters from "normal" to "congenital anomalies of the optic nerve", with all aspects of the posterior segment covered. There is a clear layout which assists in finding your way through 910 pages. There even is a guide line on how to use this book via its colour-coded chapters. Of course, this book cannot be a soft-cover handbook, since it consists of a most comprehensive collection of all kinds of retinal diseases. This book deserves its place on the shelf in your office.

Whatever retinal disease you are looking for, if you don't find it in here, you won't find it anywhere. Therefore, the index at the end of the atlas is most important and well-structured and helpful. There are many useful and well-chosen links for additional suggested reading. This latest edition has not only colour and red-free photographs, autofluorescence, fluorescein and indocyanine green angiography, but also OCT - ocular coherence tomography, and all the techniques and their pictures are colour-coded. It is the combination of these diagnostic tools which provides an almost three-dimensional impression of the pathology inside the retina. Composite photographs present an overview of more peripheral retinal pathology. My wish for a future edition is the inclusion of 200-degree wide-angle OPTOMAP SLO scans and wideangle fluorescein angiographies.

The presented array of cases and diseases is complete, and emphasizes the author's huge experience from decades of work in the field of medical retina. The name list of the contributors of cases and illustrative materials is a "Who's Who" of medical retina. And it seems as if they all contributed their most typical and best-documented cases. Over the years, and with this new edition, some cases have now been replaced by even better and more typical ones.

For all ophthalmologists involved in the making of a retinal diagnosis, this atlas is indeed mandatory and will help in diagnosing, and differentiate rare as well as common diseases. This atlas is indeed a very worthwhile investment. The intended readership is definitely not medical students, but retinal fellows who will appreciate it. In conclusion, at present I do not know of a better retinal atlas.

\footnotetext{
M. Ulbig $(\bowtie)$

Department of Ophthalmology, Ludwig-Maximilians-University,

Klinikum der Universität München,

Campus Innenstadt, Mathildenstrasse 8,

Munich D-80336, Germany

e-mail: michael.ulbig@med.uni-muenchen.de
} 